\title{
SUR LES DIVISIONS GÉNÉRIQUES DES NÉMATODES COOPERIINAE
}

\author{
(Trichostrongylidae)
}

\author{
M.-C. DURETTE-DESSET*
}

RÉSUMÉ. La présence d'une vésicule céphalique chez les Cooperiinae semble en relation avec le fait qu'ils sont également les seuls, parmi les Trichostrongylidae, à posséder un synlophe fonctionnel.

En tenant compte des modifications de cet appareil constitué fondamentalement de 5 arêtes dorsales, 5 arêtes ventrales et 3 petites paires d'arêtes latérales, orientées perpendiculairement à la paroi du corps, des hypothèses sur l'évolution du groupe sont formulées.

A partir de Chabaudstrongylus, parasite de Tragulidés et de Cervidés, le genre Cooperia a évolué dans 3 directions : a) augmentation du nombre de paires d'arêtes latérales; b) hypertrophie des arêtes ventrales par rapport aux dorsales ; c) apparition d'un axe d'orientation des arêtes, dorsoventral. Les genres Impalaia, Ortleppstrongylus (le seul parasite de Rongeurs et non de Ruminants) et Minutostrongylus ont gardé le synlophe de base et ont évolué par apparition de la monodelphie.

Par contre, chez Megacooperia, le synlophe évolue par hypertrophie des arêtes latérales, jointe à l'apparition d'un axe d'orientation ventro-dorsal chez Paracooperia.

Cooperioides est mis en synonymie de Cooperia; la validité de Minutostrongylus est reconnue.

\section{On the generic divisions in Cooperiinae (Trichostrongylidae : Nematoda).}

SUMMARY. Presence of a functional synlophe seems to be connected to presence of a cephalic vesicle in the Trichostrongyloidea and the Cooperiinae, the only members of the Trichostrongylidae with a cephalic vesicle, are also the only members with a functional synlophe.

Hypotheses concerning the evolution of the Cooperiinae are suggested on the basis of modifications of the synlophe consisting fundamentally of 5 dorsal and 5 ventral crests, and 3 pairs of small lateral crests oriented perpendicular to the body.

Arising from Chabaudstrongylus (parasites of Tragulidae and Cervidae), Cooperia has evolved in three directions: a) increase in the number of pairs of lateral crests; b) hypertrophy of ventral crests; c) appearance of a dorso-ventral axis of orientation of crests. Impalaia, Ortleppstrongylus (the only genus in the group parasitizing rodents and not ruminants) and Minutostrongylus retained the primitive synlophe and evolved in the direction of monodelphy.

Megacooperia is characterized by hypertrophy of lateral crests and Paracooperia by this as well as the appearance of a dorso-ventral axis of orientation to the synlophe.

Cooperioides is placed in synonymy with Cooperia; Minutostrongylus is recognized as valid.

* Laboratoire de Zoologie-Vers, associé au CNRS, Muséum National d'Histoire Naturelle, 61 rue Buffon, F 75231 Paris Cedex 05.

Accepté le 22 janvier 1982 
Les Cooperiinae sont les seuls Trichostrongylidae à posséder une vésicule céphalique. Le rôle de cet organe reste mystérieux. Nous pouvons noter cependant qu'il semble y avoir une corrélation entre sa présence et l'existence d'un synlophe.

Le synlophe n'est actionné par aucune musculature directe. Sa motricité paraît être sous la dépendance d'un phénomène de pression osmotique, que le Ver saurait maîtriser pour assurer sa progression et son accrochage dans l'intestin de son hôte.

On peut noter, par ailleurs, que, chez les Trichostrongyloidea, dont l'espace intra-cuticulaire est plein d'un liquide coloré en rouge, le liquide contenu dans la vésicule céphalique reste clair. Il n'y a donc pas de communication directe entre le liquide de l'espace intracuticulaire et celui de la vésicule céphalique.

Il devient ainsi possible d'imaginer que la vésicule céphalique est un organe qui enregistre les variations de pression osmotique du milieu dans lequel se trouve le Ver et que ces différences de pression sont enregistrées par les amphides (cf. Wright, 1975).

Quoi qu'il en soit, la présence d'une vésicule céphalique chez les Cooperiinae laisse supposer que le synlophe est fonctionnel et que, contrairement aux autres Trichostrongylidae, il devient possible d'en tenir compte dans l'évolution et la classification des genres.

Les travaux de Gibbons, 1977, 1978 a, b, de Gibbons et Khalil, 1980, et de Khalil et Gibbons, 1976, mettent précisément en évidence la complexité de cet appareil chez un grand nombre de Cooperiinae.

Plusieurs arguments nous amènent à penser que le synlophe primitif d'un Cooperiinae comporte 5 arêtes dorsales, 5 arêtes ventrales et 3 paires de petites arêtes latérales, orientées perpendiculairement à la paroi du corps.

a) Ce synlophe est celui de l'espèce-type du genre Chabaudstrongylus DuretteDesset et Denké, 1978, qui, avec sa côte dorsale longue et peu profondément divisée, apparaît comme le plus primitif des Cooperiinae.

b) Chabaudstrongylus est parasite de Tragulidés et de Cervidés ${ }^{1}$. A l'exception d'Ortleppstrongylus Durette-Desset, 1970, parasite de Rongeurs, tous les autres Cooperiinae se rencontrent chez les Tauroidea (Bovidés - Giraffidés). Chabaudstrongylus est donc le seul Cooperiinae dont le spectre d'hôtes recouvre des hôtes primitifs.

c) On sait, par ailleurs, que, chez les Nématodes parasites d'animaux, lorsqu'il existe un dimorphisme sexuel, le mâle a des caractères plus primitifs que ceux de la femelle. Chez les Cooperiinae, il existe parfois un dimorphisme sexuel dans le nombre de paires latérales du synlophe. Dans ce cas, ce nombre est plus proche du chiffre 3 chez le mâle que chez la femelle. C'est l'inverse chez $C$. okapi, où la femelle possède 3 paires d'arêtes et le mâle 2 , mais, chez cette espèce, le diamètre du corps de la femelle est nettement plus grand que celui du mâle.

I. Nous classons dans le genre Chabaudstrongylus C. dubosti (Durette-Desset et Chabaud, 1974), parasite d'un Tragulidé éthiopien, C. caballeroi (Durette-Desset et Krishnasamy, 1976) n. cb. (= Cooperia caballeroi Durette-Desset et Krishnasamy, 1976), parasite d'un Tragulidé en Malaisie, dont le synlophe est composé de 7 arêtes dorsales, 7 arêtes ventrales et 3 paires de petites arêtes latérales, et $C$. ninhae (Drozdz, 1967) n. cb. (=Cooperia ninhae Drozdz, 1967), parasite du Muntjac au Viet-Nam, dont le synlophe n'est pas connu. 
d) Il est intéressant de remarquer que le synlophe de Chabaudstrongylus ne diffère pas de celui de certaines espèces du genre Molineus. Il a été constaté chez les Molineides que les synlophes, tout en conservant la symétrie bilatérale primitive, sont très polymorphes. Il semble y avoir eu des tentatives évolutives variées, dont certaines seulement sont retenues par la sélection et aboutissent à de véritables lignées évolutives (cf. Durette-Desset, 1973, 1979, Durette-Desset et Chabaud, 1975).

La diversification du synlophe en différentes lignées évolutives, telle qu'elle est observée chez les Molineidae, se rencontre également chez les Cooperiinae.

Dans le seul genre Cooperia Ransom, 1907, qui semble directement issu de Chabaudstrongylus, nous observons ainsi plusieurs " tentatives évolutives " :

a) Augmentation du nombre des petites arêtes latérales : 5 paires : $C$. verrucosa Mönnig, 1932 et $C$. neitzi Mönnig, 1932.

b) Diminution du nombre des petites arêtes latérales : 3 paires : C. okapi q Leiper, 1935; 2 paires : C. okapi ô; C. oncophora Railliet, 1898 ; C. pectinata Ransom, 1907 ; C. punctata (Linstow, 1906) ; C. rotundispiculum Gibbons et Khalil, 1980 ; C. yoshidai Mönnig, 1939 ; une paire : C. hamiltoni Mönnig, 1932, C. hungi Mönnig, 1931 ; zéro paire : C. antidorca Mönnig, 1931, C. chabaudi Diaouré, 1964, C. hepaticae (Ortlepp, 1938) n. cb. (= Cooperioides hepaticae Ortlepp, 1938).

Chez toutes ces espèces, il y a conservation des arêtes dorsales et ventrales primitives.

c) A partir du type précédent, on observe une spécialisation des arêtes ventrales, qui deviennent plus grandes que les dorsales chez $C$. curticei (Giles, 1892) et $C$. fuelleborni Hung, 1926 (avec 2 paires de petites arêtes latérales).

Enfin, il y a apparition d'un axe d'orientation dorso-ventral des arêtes (avec 1 paire de petites arêtes latérales) chez le ô de $C$. connochaeti Boomker, Horak et Alves, 1979, sans petites arêtes latérales chez la .

L'évolution des autres Cooperiinae se fait également à partir de Chabaudstrongylus, par spécialisations diverses :

- Genres Impalaia Mönnig, 1923, Ortleppstrongylus et Minutostrongylus Le Roux, 1936. Le synlophe est de type Chabaudstrongylus, mais la taille des petites arêtes latérales tend à devenir égale à celle des autres arêtes. Ils sont tous trois monodelphes.

Ortleppstrongylus, parasite de Rongeurs et non de Ruminants, se distingue d'Impalaia par une côte dorsale moins longue et des côtes 8 plus longues que la côte dorsale.

Minutostrongylus a généralement été considéré comme synonyme d'Impalaia (cf. Gibbons, Durette-Desset et Daynes, 1977, Boomker, 1977). En fait, il peut être séparé des 2 autres genres, d'une part par sa dorsale beaucoup plus courte, d'autre part, par la position de la côte 4. Cette côte n'est pas accolée à la côte 5 et sa papille est située plus près de celle de la côte 3 que de la côte 5 , ce qui est l'inverse chez les 2 autres genres. Nous savons, par ailleurs, par l'étude des Molineinae (cf. DuretteDesset et Chabaud, 1980), que cet élément revêt une grande importance systématique. 
Contrairement aux genres précédents, où le synlophe reste de type primitif, le synlophe des deux genres suivants a évolué par hypertrophie des arêtes latérales (genre Megacooperia Khalil et Gibbons, 1976), puis apparition d'un axe d'orientation ventro-dorsal (genre Paracooperia Travassos, 1935).

Megacooperia se distingue en outre par l'allongement considérable du lobe dorsal ; Paracooperia par une indentation caractéristique des spicules.

- Cooperioides Daubney, 1933, espèce-type C. hamiltoni (Mönnig, 1932), est mis en synonymie de Cooperia, car le niveau de bifurcation de la côte dorsale est variable chez les Cooperia s. $s$. et ne permet pas de séparer deux groupes distincts.

- Schwartziella Le Roux, 1936, espèce-type S. nodulosa (Schwartz, 1928), a été transféré dans le genre Paracooperia par Travassos, 1937, et la synonymie admise par tous les auteurs.

- De la même façon, Anthostrongyhus Croveri, 1929, espèce-type A. somalilensis Croveri, 1929, a été mis en synonymie d'Impalaia par Travassos, 1937.

\section{BIBLIOGRAPHIE}

Boomker J. : A revision of the genus Impalaia Mönnig, 1924. Onderstepoort J. vet. Res., 1977, 44, I3I-I 38 .

Boomker J., Horak I. G., Alves R. : Cooperia connochaeti sp. nov. (Nematoda, Trichostrongylidae) from the blue wildebeest Connochaetes taurinus (Burchell, 1823). Onderstepoort J. Vet. Res., 1979, 46, 83-86.

Croveri F. : Un nuovo strongylide : Anthostrongylus somalilensis Croveri, I9I7 (n.g. n.s.). Parasita dei dromadari somali e la strongylosi intestinale epizootica dei dromadari "Ber Cursu ". Arch. Ital. S. Med. Colon. Tripoli, 1929, 10, 143-170.

Daubney R. : Trichostrongylid Nematodes from sheep in Kenya. Parasitol. (Cambridge), 1933, $25,224-24 \mathrm{I}$.

Diaoure A. : Strongylides parasites de Mammifères du Congo-Brazzaville. Ann. Parasitol., 1964,

39, $243-284$.
DRozDZ J. : "Cooperia ninhae sp. n. (Nematoda : Trichostrongylidae), a parasite of Muntjacus muntjak in Vietnam ». Acta parasit. pol., 1967, 14, 209-211.

Duretre-Desset M.-C. : Affinités de l'Héligmosome Ortleppstrongylus bathyergi (Ortlepp, 1939) n. gen., n. comb. avec les Trichostrongylides Molineinae. Bull. Mus. nat. Hist. nat., $2^{\mathrm{e}}$ sér., I970, 42, 4I 5-4I8.

DURETTE-DESSET M.-C. : Nématodes Trichostrongyles du genre Molineus Cameron, 1923, parasites d'Insectivores malgaches. Ann. Parasitol. Hum. Comp., 1973, 48, 677-698.

Durette-Desset M.-C. : Les Nematodirinae (Nematoda) chez les Ruminants et chez les Lagomorphes. Ann. Parasitol. Hum. Comp., 1979, 54, 313-329.

Duretre-Desset M.-C., Chabaud A. G. : Trois nouveaux Nématodes parasites du Chevrotain aquatique : Hyemoscus aquatiqus au Gabon (collection G. Dubost). Bull. Mus. nat. Hist. nat., $3^{\mathrm{e}}$ sér., 1974, no 205, Zool. I35, 75-87.

Duretre-Desset M.-C., Chabaud A. G. : Nématodes Trichostrongyloidea parasites de Microchiroptères. Ann. Parasitol. Hum. Comp., I975, so, 303-337.

DURETTE-Desset M.-C., KrishNasamy M. : Cooperia caballeroi n. sp. Nematoda Trichostrongylidae, parasite de Tragulus javanicus en Malaisie. Excerta Parasit. Mem. Dr. E. Caballero y Caballero, Pub. espec. Inst. Biol. Mexico, 1976, 429-432.

GibBons L. M. : Revision of the genus Paracooperia Travassos, 1935 (Nematoda : Trichostrongylidae). J. Helminthol., 1978, s2, $231-249$.

GrbBons L. M. : Revision of the genus Cooperioides Daubney, I933 (Nematoda : Trichostrongylidae), J. Helminthol., 1978, 52, 311-322.

Gibbons L. M. : Revision of the african species of the genus Cooperia Ransom, I907 (Nematoda, Trichostrongylidae). Systematic Parasitology, 1981, 2, 219-252.

Gibbons L. M., KhaliL L. F. : Some new Trichostrongylid nematodes from east african artiodactylids. Systematic Parasitology, 1980, $1,9 \mathrm{I}$-104.

Giles G. M. J. : On a new Sclerostome from the large intestine of Mules. A description of two new Nematodes parasites found in sheep. Scient. Mem. Med. Officers Army India, 1892, 7, 25-30. 
Hung S. L. : Utber zwei neue parasitische Nematoden, Cooperia fuelleborni aus dem wasserbock und Oesophagostomum maurum aus dem Mohrenmakak. Arch. Schiffs. Tropen Hyg., 1926, $30,42 \mathrm{I}-430$.

KhaLIL L. F., GibBons L. M. : The helminth parasites of the suni, Nesotragus moschatus von Dueben, 1846 from Kenya with the description of a new genus and two new species of nematodes (Vermes). Rev. Zool. afr., 1976, 90, 559-577.

Leiper R. T. : Helminth parasites obtained from the okapi at post-mortem. Proc. Zool. Soc. London, 1935, 4, 949.

LE Roux P. L.: A new Trichostrongylid (Minutostrongylus taurotragi n.g. n. sp.) of the subfamily Heligmosominae from an African antelope. J. Helminthol., 1936, 14, 73-76.

Mönnig H. O. : On some new South African parasitic nematodes. Trans. R. Soc. S. Africa, 1923, II, I05-II 7 .

Mönnig H. O. : Wild antelopes as carriers of Nematode parasites of domestic ruminants. Part II. I8th Rep. Dir. Vet. Serv. Ass. Indust., 1932, 153-172.

MönNig H. O. : Wild antelopes as carriers of Nematodes parasitic in domestic ruminants. Onderstepoort J. Pretoria, 1933, I, 77-92.

MöNnig H. O.: Cooperia yoshidai $\mathrm{n}$. sp., a nematode parasite of the redbuck, Redunca arundinum. Vol. Jubilar Prof. Yoshida, 1939, 29I-294.

ORTLEPP R. J. : South African helminths. Part V. Some avian and mammalian helminths. Onderstepoort J. Pretoria, 1938, II, 63-104.

RANSOM B. H. : Notes on parasitic Nematodes including descriptions of new genera and species and observations on life histories. Bull. U.S. Dept. Agric. Anim. Indust. Circ., 1907, 116, 52 p.

Travassos L. : Alguns noves generos e especies de Trichostrongylidae. Rev. med. cirurgica do Brasil, I935, 43, II, 345-36I.

Travassos L. : Revisao da familia Trichostrongylidae Leiper, I912. Monogr. Inst. O. Cruz, I937. $I, 5 \mathrm{I} 2 \mathrm{p}$.

WRIGHT K. A. : Cephalic sense organs of the rat hook worm, Nippostrongylus brasiliensis form and function. Can. J. Zool., I975, 53, I1 $31-1146$. 